This item was submitted to Loughborough's Research Repository by the author.

Items in Figshare are protected by copyright, with all rights reserved, unless otherwise indicated.

\title{
Individual differences in kindergarten math achievement: the integrative roles of approximation skills and working memory
}

PLEASE CITE THE PUBLISHED VERSION

http://dx.doi.org/10.1016/j.lindif.2013.09.012

\section{PUBLISHER}

(C) Elsevier Inc.

\section{VERSION}

AM (Accepted Manuscript)

\section{PUBLISHER STATEMENT}

This work is made available according to the conditions of the Creative Commons Attribution-NonCommercialNoDerivatives 4.0 International (CC BY-NC-ND 4.0) licence. Full details of this licence are available at: https://creativecommons.org/licenses/by-nc-nd/4.0/

\section{LICENCE}

CC BY-NC-ND 4.0

\section{REPOSITORY RECORD}

Xenidou-Dervou, Iro, Bert De Smedt, Menno van der Schoot, and Ernest C.D.M. van Lieshout. 2019.

"Individual Differences in Kindergarten Math Achievement: The Integrative Roles of Approximation Skills and Working Memory". figshare. https://hdl.handle.net/2134/20085. 
Individual Differences in Kindergarten Math Achievement: The Integrative Roles of Approximation Skills and Working Memory.

Iro Xenidou-Dervou ${ }^{\mathrm{a}^{*}}$, Bert De Smedt ${ }^{\mathrm{b}}$, Menno van der Schoot ${ }^{\mathrm{a}}$, Ernest C. D. M. van Lieshout $^{\mathrm{a}}$

${ }^{\text {a }}$ Department of Educational Neuroscience, Faculty of Psychology and Education, VU University Amsterdam, the Netherlands

${ }^{\mathrm{b}}$ Faculty of Psychology and Educational Sciences, University of Leuven, Belgium

*Corresponding author at:

Dept. of Educational Neuroscience, Faculty of Psychology and Education, VU University Amsterdam, Post address: Van der Boechorststraat 1, 1081 BT, Amsterdam, the Netherlands. Tel.: +31 (0)20 5989413; E-mail address: I.Xenidou-Dervou@,vu.nl (I. Xenidou-Dervou) 


\begin{abstract}
Kindergarteners can conduct basic computations with large nonsymbolic (e.g. dots, objects) and symbolic (i.e. Arabic numbers) numerosities in an approximate manner. These abilities are related to individual differences in mathematics achievement. At the same time, these individual differences are also determined by Working Memory (WM). The interrelationship between approximation, WM and math achievement has been largely unexplored. Also, the differential role of nonsymbolic and symbolic approximation in explaining math competencies is yet unclear. We examined an integrative theoretical model on the association of approximation (addition and comparison) and WM in 444 kindergarteners. As expected, approximation entailed two distinct abilities (nonsymbolic and symbolic). Both abilities correlated with mathematics achievement (i.e. counting and exact arithmetic), even when WM was taken into account. The association between nonsymbolic approximation and math achievement was completely mediated by symbolic approximation skills. These findings add to our understanding of the cognitive architecture underlying kindergarten math achievement.
\end{abstract}

Keywords: Numerical cognition; Nonsymbolic Approximation; Symbolic Approximation; Working Memory; Math Achievement. 


\section{Introduction}

From the girl, who realizes she was given less candies than her older sister, to the adult at the counter, who estimates his change, math is everywhere. There are large differences between individuals in the way they develop mathematical competencies (Geary, 2011). Research so far has been very fruitful in identifying math-specific (neuro)cognitive precursors for mathematical achievement at the early stages of development (see Dehaene, 2011; Feigenson, Dehaene, \& Spelke, 2004; Piazza, 2010). Crucial roles have been attributed to children's abilities to conduct simple computations in an approximate manner with large nonsymbolic (e.g. objects, dots, sounds), and symbolic (i.e., Arabic numbers) numerosities (e.g. Barth, La Mont, Lipton, Dehaene, Kanwisher, \& Spelke, 2006; Gilmore, McCarthy, \& Spelke, 2007; 2010; Libertus, Feigenson, \& Halberda, 2011). However, the differential role that nonsymbolic and symbolic approximate magnitude skills play in the early development of children's math proficiency and deficiency is an ongoing discussion in the literature (e.g. Gilmore, et al., 2010; Holloway \& Ansari, 2009; Mundy \& Gilmore, 2009; Noël \& Rouselle, 2011).

Previous research in the domain has mostly been conducted with small populations limiting the generalizability of their results. Also, few have focused on the kindergarten age, i.e. before the start of formal primary education, or have used multiple measures of approximation skills. Finally, the effect of the domain-general capacity of Working Memory (WM), which has been demonstrated to affect approximation processing (Caviola, Mammarella, Cornoldi, \& Lucangeli, 2012; Xenidou-Dervou, Van Lieshout, \& Van der Schoot, in press) has not yet been accounted for in related research.

The present study tried to address these issues by investigating how nonsymbolic and symbolic approximation skills were related to kindergarten math achievement when taking 
into account WM capacity. We conducted a large-scale correlational study that allowed us to examine the factorial structure and interrelation of the aforementioned cognitive skills. With this design, we were able to address the issue of which specific role nonsymbolic and symbolic approximation skills (addition and comparison) could play in explaining individual differences in math achievement at the kindergarten age.

\subsection{Nonsymbolic and symbolic approximation skills}

Learning addition in the form of $\mathrm{a}+\mathrm{b}=\mathrm{c}$, is a strenuous process and its mastery may take years within primary school instruction (Ashcraft \& Fierman, 1982; Haman \& Ashcraft, 1985). Nonetheless, kindergarteners appear to perform easily above chance level in addition tasks that call for an approximate response (Barth, La Mont, et al., 2006; Gilmore et al., 2007; 2010). The literature assumes the existence of an evolutionary ancient system, the so-called Approximate Number System (ANS), with which humans and animals are enriched in order to estimate stimuli in nature (see Brannon, Jordan, \& Jones, 2010; Feigenson et al., 2004; Piazza, 2010). Preschool children, before having acquired formal school instruction, have been consistently demonstrated to be able to successfully compare and add approximately large quantities of nonsymbolic stimuli (Barth, et al., 2006; Gilmore, et al., 2010; Libertus, et al., 2011; Mazzocco, Feigenson, \& Halberda, 2011; Xenidou-Dervou, et al., in press). They can do so even when these quantities are presented in different modalities and formats (Barth, Beckmann, \& Spelke, 2008; McNeil, Wagner Fuhs, Keultjes, \& Gibson, 2011). Performance in tasks assessing these skills is characterized by the so-called ratio effect. The more the ratio difference between two target numerosities approaches 1 , the harder it is to compare them (Barth et al., 2006; Izard \& Dehaene, 2008). Additionally, research has shown that nonsymbolic approximate addition is as accurate as nonsymbolic approximate comparison, which calls only for the comparison of two numerosities (Barth et al., 2006). The aforementioned skills are referred to here as nonsymbolic approximation skills. 
Five-year old children are able to solve corresponding approximate comparison and addition problems even with symbolic stimuli when they have not actually been formally taught large-numerosity symbolic arithmetic (Gilmore, et al., 2007; McNeil et al., 2011). These abilities are referred to as symbolic approximation skills and they are subject to the same characteristic effects as nonsymbolic approximation (Gilmore et al., 2007). This shared cognitive profile suggested that children recruit their ANS representations when engaging in symbolic approximation. In other words, it is assumed that symbolic approximate representations are mapped onto and are fostered by one's preexisting nonsymbolic representations. More precise mapping representations between nonsymbolic and symbolic numerosities have been associated with better mathematical achievement in young children (Mundy \& Gilmore, 2009). In this respect, the approximation system is sometimes viewed as a single system.

The precise role that nonsymbolic approximation plays in the process of acquiring formal mathematical knowledge and skills is yet unclear. Some assume that it comprises the foundation for learning symbolic arithmetic, i.e. as taught in school (Barth et al., 2006; Gilmore et al., 2007; Halberda et al., 2008; Halberda \& Feigenson, 2008; Libertus, et al., 2011; Mazzocco, et al., 2011; Mundy \& Gilmore, 2009). In line with this view, Gilmore et al. (2010) found preschoolers' nonsymbolic approximation skills to be predictive of their counting and mathematical skills. This occurred irrespective of the children's verbal intelligence and reading skills.

Others, however, support that nonsymbolic approximation skills are not determinant for early mathematics achievement (LeCorre \& Carrey, 2007; Noël \& Rouselle, 2011). It is assumed that the skills in question become important only after the age of eight years (Noël \& Rouselle, 2011). This assumption is supported by findings, where nonsymbolic task performance failed to account for individual differences in early age math achievement (e.g. 
De Smedt \& Gilmore, 2011; Mundy \& Gilmore, 2009; Holloway \& Ansari, 2009). Instead, nonsymbolic abilities played an important role for children of older ages (Piazza et al., 2010; Mazzocco et al., 2011). As outlined by Noël and Rouselle, (2011), the age factor is crucial. The aforementioned studies comprised children from six years of age and older. The critical age of kindergarten, however, has received less attention. Also, few studies have made use of multiple approximation measures. Sasanguie, De Smedt, Defever, and Reynvoet's (2011) cross-sectional study, tested kindergarteners in nonsymbolic and symbolic comparison and number line tasks. They found a strong association between symbolic but not nonsymbolic magnitude comparison with kindergarteners' math achievement. The small population sample used in this study, however, restricts the generalizability of the results. Furthermore, one may wonder as to how validly number line tasks measure numerical representation because:

“Number line estimation tasks assess only one aspect of children's numerical representations, namely, the linearity of children's symbolic representations. Tasks involving more general nonsymbolic representations are necessary to fully investigate the role of this system in mathematics learning." (Mundy \& Gilmore, 2009; pp. 492). Inspired by Barth and colleagues' (2005; 2006) and Gilmore and colleagues' (2007) measures, the present correlational study assessed nonsymbolic and symbolic approximation skills in comparison as well as in addition, in a large population sample of kindergarteners. This design permitted the usage of structural equation modeling techniques that could determine the latent structure and interrelation of the targeted cognitive systems; namely, approximation skills, WM and math achievement.

\subsection{The role of $W M$}

WM refers to the cognitive system that is dedicated to the short-term storage, regulation and manipulation of information in an online manner (Baddeley, 2003). Baddeley's multicomponent model of WM (Baddeley, 1986; 2003) incorporates a master system, the central executive $(\mathrm{CE})$, which controls, monitors and regulates the processes of two auxiliary 
systems; the phonological loop (PL) and the visuospatial sketchpad (VSSP). These "slave" subsystems are responsible for the temporary storage of phonological and visuospatial information respectively. All WM components have been shown to play a role in math performance. The extent of involvement of each component is dependent upon the different requirements of a given task (Noël, 2009; Rasmussen \& Bisanz, 2005; Simons, Willis, \& Adams, 2012; for a review see DeStefano \& LeFevre, 2004).

In general, there is compelling evidence surrounding the importance of WM in explaining individual differences in mathematical achievement (Bull, Espy, \& Wiebe, 2008; De Smedt et al., 2009a; DeStefano \& LeFevre, 2004; LeFevre, DeStefano, Coleman, \& Shanahan, 2005; Passolunghi, Vercelloni, \& Schadee, 2007; Raghubar, Barnes, \& Hecht, 2010). More specifically, it has been strongly related to both counting (Geary, Hoard, ByrdCraven, \& DeSoto, 2004) as well as mental arithmetic (for a review see DeStefano \& LeFevre, 2004). Recent findings addressed the association between children's approximation skills and WM. With a dual-task study, Xenidou-Dervou, et al. (in press) demonstrated that the $\mathrm{CE}$ is necessary for nonsymbolic approximate addition processing in kindergarten. On the other hand, Caviola et al. (2012) showed that children's symbolic approximate addition called for PL or VSSP resources according to the demands and constraints of a task. Thus, various WM components are necessary for processing different approximation problems. In essence, these findings suggest that individual differences in approximation skills can be explained by individual differences in WM capacity. To our knowledge, however, the effect of this interrelationship on kindergarteners' math achievement has been unexplored.

\subsection{The present study}

In kindergarten, math achievement involves children learning how to count and beginning to understand the basic principles underlying addition (e.g. Geary, 2011). At this 
developmental stage counting skills form children's basis for learning how to conduct their first simple additions and constitute a measure of their math achievement (Geary, 2011; Geary, et al., 2004). We addressed the question: How are kindergarteners' approximation and WM skills associated with these mathematical competencies? To the best of our knowledge, this study is the first to take into accumulating account the aforementioned skills in the process of math learning.

Amassing the findings presented in the previous sections, we formulated a comprehensive model on the integrative relationship of approximation, WM and math achievement in kindergarten age. As illustrated in Figure 1, we hypothesized that: (1) Nonsymbolic and symbolic approximation skills would comprise two distinct abilities (Holloway \& Ansari, 2009); (2) WM, as a domain-general cognitive ability, would predict math achievement beyond the effect of the domain-specific cognitive abilities (DeStefano \& LeFevre, 2004; Geary, 2011; LeFevre et al., 2005; Raghubar, Barnes, \& Hecht, 2010); (3) WM would influence performance in both nonsymbolic (Xenidou-Dervou et al., in press) and symbolic approximation (Caviola et al., 2012); (4) Nonsymbolic approximation would predict symbolic approximation since the latter is assumed to recruit nonsymbolic approximate representations (Gilmore et al., 2007), and lastly that (5) Nonsymbolic (Gilmore, et al., 2010) and symbolic approximation (De Smedt \& Gilmore, 2011) would predict kindergarteners’ mathematical achievement.

- Insert Figure 1 -

Hypotheses 4 and 5 suggested that symbolic approximation partially mediates the relationship between nonsymbolic approximation and mathematical achievement (Figure 1). 
Because the role of approximation skills in the process of learning mathematics remains elusive (Gilmore et al., 2010; Mundy \& Gilmore, 2009; Noël \& Rouselle, 2011), we tested two additional models. Beyond the partial mediation pattern, we examined whether a model with only the direct effects of nonsymbolic and symbolic approximation on math achievement, or a model where the effect of nonsymbolic approximation is completely mediated by symbolic approximation would best fit the data.

\section{Method}

\subsection{Participants}

Participants were 444 kindergarten children (199 girls and 245 boys) with mean age $5.59, S D=0.35$, years. Six children did not undertake the complete set of tasks: One child changed schools and the other five were unavailable on a testing day due to illness (see Table 1). The data described in this paper were collected as part of an interlinked project entitled the "XXXX" (see www.XXXX). We contacted regular schools from all around the Netherlands and recruited kindergarteners from 25 of these schools. Written informed consent forms were acquired from all children's legal guardians. The number of participating children from each school ranged from 3 up to 59. We included kindergarteners from the whole ability range. $95.3 \%$ of the children had Dutch nationality. All children spoke Dutch and, according to teacher reports, $5.8 \%$ of the children spoke a second language. Participants were from middleto high-class SES community environments. More than $40 \%$ of the children's parents had completed higher levels of education (in the Dutch educational system: HBO and higher levels; fathers: $44.4 \%$, mothers: $41.3 \%$ ).

\footnotetext{
${ }^{1}$ This information has been omitted for the blind-review process
} 


\subsection{Procedure}

Children were tested individually in a quiet room within the school facilities during regular school hours by trained experimenters. The data reported regard a subset of tasks that were collected as part of the $\mathrm{XXXX}^{1}$. Testing for the measurement wave began in November and ended in January of the same academic year. It included three testing sessions with each child. The tasks reported were included within the first and the second session, each lasting approximately 30 minutes. Consecutive sessions took place within a maximum time-span of two weeks and a minimum of one day. Participants received stickers after each session in order to sustain their interest and motivation.

\subsection{Materials}

All tasks used in this study were computerized and presented with E-Prime, version 1.2 (Psychological Software Tools, Pittsburgh, PA, USA). HP Probook 6550b type laptops were utilized with the screen resolution set to $600 \times 480$ pixels.

2.3.1 Nonsymbolic and symbolic approximation. Four tasks were used to assess children's nonsymbolic and symbolic approximation skills: two addition tasks (nonsymbolic and symbolic) and two comparison tasks (nonsymbolic and symbolic).

2.3.1.1 Nonsymbolic approximate addition. We used an adapted version of the dottask described in Xenidou-Dervou et al. (in press; adapted from Barth et al. 2005; 2006). Images of a boy on the far top left side of the screen (Peter) and a girl on the far top right side of the screen (Sarah) were added. Thus, a small story was integrated in the task description about Peter and Sarah receiving an amount of marbles (dots). In each trial, the child was asked to indicate whether Sarah or Peter received the most marbles. This task entailed 6 practice and 24 testing trials. A trial entailed four sequential steps: 1) a set of blue dots (marbles) dropped down on the left side of the screen, 2) these dots were covered up by a grey 
box, 3) another set of blue dots dropped into the box, 4) on the right top side of the screen, a set of red dots popped out and then dropped down. The duration of each of these animated steps was $1300 \mathrm{~ms}$ and between each step there was an interval of $1200 \mathrm{~ms}$. This fast interchange of events prevented children from counting the dots. Children could respond from the moment the red dots popped up on the right upper side of the screen. Then, they had a maximum of $7000 \mathrm{~ms}$ to respond. There was a $300 \mathrm{~ms}$ interval before the next trial started. Numerosities in this task ranged from 6 to 70. The sum of the blue arrays differed from the comparison red set by three ratios 4:7, 4:6, 4:5 (easy, middle and difficult ratio level; 8 trials each). In half of the trials the sum of the blue sets was larger than the red comparison set. In the other half, the comparison target was larger. Testing trials were designed in a manner that allowed post-hoc examination of whether children made use of any strategies alternative to approximation (see Appendix, Table A1). We controlled for responses being reliant on the physical features of the dot-sets (continuous quantity variables) by controlling for dot size, total surface area, total contour length and density (Barth et al., 2006; Gilmore et al., 2010). All dot-stimuli were developed with MATLAB 7.5 R2007b.

Children were instructed to respond as correctly and as fast as possible. They responded by pressing the corresponding response button in front of them (blue or red). Instructions were given verbally during practice. No feedback was provided during the testing trials except for verbal encouragement to sustain engagement with the task. Before initiating the task, the experimenter would ask the child to point out on the screen the set of blue and the set of red dots in order to identify possible difficulties related to colorblindness. None of the children in our study indicated any difficulties with distinguishing the two colors.

2.3.1.2 Nonsymbolic approximate comparison. This task was similar to the previous one with the key difference of not having two blue sets of dots dropping in the grey box but 
only one set: the sum of the original two (see Appendix, Table A1). Children were now asked to compare this blue array of dots with the subsequently presented red array.

2.3.1.3 Symbolic approximate addition. This task was also based on the nonsymbolic approximate addition task. In this condition, dot-sets were replaced with blue or red boxes that carried the Arabic numeral of the corresponding numerosity.

2.3.1.4 Symbolic approximate comparison. This task was the same as the nonsymbolic version except that Arabic numbers instead of dots had to be compared.

2.3.2 WM. The Dutch version of four tasks (Toll, Van der Ven, Kroesbergen \& Van Luit, 2011; Messer, Leseman, Boom, \& Mayo, 2010) from the Automated WM Assessment test battery (AWMA; Alloway, 2007) were used to assess children's capacity on the three components of WM. These tasks have been widely used and have been shown to have high reliability (Alloway et al., 2006). Each task started with a short practice session. Instructions were read aloud by the experimenters.

2.3.2.1 Phonological Loop (PL). The Word recall forward task was used to tap children's PL capacity. In this task, the child heard a sequence of letters and had to repeat them in the presented order. A correct response necessitated that the child would repeat the words correctly and in the right order. Task difficulty ranged from one up to five words. If the children gave four consecutive correct responses they moved to the next difficulty level. If they gave three consecutive incorrect answers, the task was automatically terminated.

\subsubsection{Visuospatial sketchpad (VSSP). The Cross Matrix was used to assess} children's VSSP capacity. This task corresponds to the Dot Matrix task of the AWMA battery. In this version, crosses were presented instead of dots in order to avoid confounding aspects with our nonsymbolic tasks. The child saw an empty $4 \times 4$ matrix and was asked to point to the box where the cross had appeared earlier. Across trials, the level of difficulty ranged from the presentation of one cross up until a sequence of five crosses appearing in the 
matrix. When a child responded correctly on four consecutive trials within a block, he/she was automatically upgraded to the next difficulty block. Three consecutive incorrect responses lead to the termination of the task. A response was automatically registered as incorrect: a) when one of the boxes was not indicated, b) when the sequence of the boxes the child pointed out was wrong, and lastly c) when a box was recalled incorrectly.

2.3.2.3 Central Executive (CE). Repovš and Baddeley (2006) highlighted the fractionation of the CE into a phonological and a visuospatial subsystem based on the type of information that is being manipulated. Therefore, we used a phonological (Word recall backwards) and a visuospatial (Odd One Out) CE task.

2.3.2.3.1 Word Recall Backwards. The procedure was similar to that of the word recall forward apart with the difference that the child was now required to recall the words backwards. Difficulty ranged from a series of two up to six words. Correctness of responses was based on whether the child repeated correctly the words in the right sequence.

2.3.2.3.2 Odd One Out. The child saw a set of three boxes one next to each other. Each box contained a shape and one shape was different from the other two. The child had to point at the odd shape. Then a new set of shapes was presented. More difficult trials contained more sets of shapes. This task entailed five levels of difficulty. At the end of each trial, three empty boxes were presented, where the child had to point to the locations of the previously shown odd shapes in the correct order. A response was correct when both the locations and the order were recalled correctly. When a child responded correctly to three consecutive trials of the same length, the sequence increased by one. After two consecutive incorrect responses, the task was terminated.

\subsubsection{Math Achievement.}


2.3.3.1 Counting. Children's counting skills were measured with the relevant subscales from the Early Numeracy Test - Revised (ENT-R; Van Luit \& Van de Rijt, 2009). The ENT-R is a test used for the assessment of children's (aged $4-7$ years) preparatory mathematical abilities. The original ENT-R consists of nine subscales, each with five items. Two analogous versions of this test exist (A and B). For this study, we used the four subscales of the A version (20 items), which assessed children's counting skills and their ability to apply their knowledge of the counting system, namely: 1) Using number words, i.e., counting forwards and backwards up to maximum twenty; 2) Structured counting, i.e., counting while pointing to objects; 3) Resultative counting, i.e., counting without pointing to objects; 4) General understanding of numbers and using the counting system in everyday life. On a given item, a correct response was scored with one point and an incorrect with zero. The counting items of the ENT-R demonstrated high reliability: Cronbach's alpha $=.82$.

2.3.3.2 Exact Symbolic Addition. An adapted version of the Jenks, De Moor and Van Lieshout (2009) addition task was used. It entailed 15 problems in the form of $a+b=c$, where "a" and "b" were not equal and both larger than 1. After one practice trial, the child received 10 items with simple $(\mathrm{c}<10)$ and 5 items with harder addition problems $(10<\mathrm{c}<$ 16). Each item appeared on the monitor and remained until the child gave a verbal response. Children were instructed to respond as correctly and as fast as possible. The experimenter pressed the space bar the moment the child responded recording his/her response time (RT). Because accuracy on this task was, as expected, low and many children (78/444) scored 0 , RTs were not used in this study. The items of this task's adapted version demonstrated a high level of internal consistency, Cronbach's alpha $=.91$.

\subsection{Analyses}


Our results section entails four parts. In the first part, we report preliminary analyses, where we examined the effectiveness of the nonsymbolic and symbolic approximation tasks. The second part entails descriptive statistics on all the measures assessed and includes evaluation of their appropriateness for the subsequent Confirmatory Factor Analyses (CFA) and Structural Equation Modeling (SEM). Thirdly, we report the results of two CFA analyses (measurement models), which determined the factor structure of our measures as representatives of three (CFA1: WM, approximation, math achievement) or four latent variables (CFA2: WM, nonsymbolic approximation, symbolic approximation, math achievement). Children's scores on the Cross Matrix, Odd One Out, Word Recall Forward and Word Recall Backwards were set as indicators of the exogenous latent variable WM. Participants' performance on counting and exact symbolic addition were set as indicators of the math achievement latent variable. In CFA1 the four approximation measures indicated a single approximation latent variable. In CFA2 nonsymbolic approximate addition and comparison indicated the nonsymbolic approximation latent variable. Similarly, symbolic approximate addition and comparison indicated the symbolic approximation variable. In the fourth part of our results, SEM analyses were conducted to examine the structural model associations between the latent variables.

CFA and SEM analyses were conducted using the Mplus analytic software, version 6.11 (Muthén \& Muthén, 1998-2011). SEM allows for the statistical evaluation of adequacy of a set of assumed regression (single-headed arrows) or correlation (double-headed) equations between the latent variables based on the correlation matrix of the observed variables (Hoyle, 1995). Among other advantages, SEM modeling is distinct from path or simple regression modeling because it distinguishes and controls for two different types of measurement errors: errors on the level of the observed variables and errors on the level of the structural equations (paths). Maximum-likelihood estimation procedures (MLE) were used. 
Graphical model representations depict the standardized solutions of the corresponding models.

We tested five models: a) a measurement model with 3 factors, b) a measurement model with 4 factors, c) a model that assumes a partial mediation effect of symbolic approximation on the relationship between nonsymbolic approximation and math achievement (Partial Mediation model), d) a model where only the direct effects of nonsymbolic and symbolic approximation on math achievement were assumed (Direct Effects model) and e) a model where the effect of nonsymbolic approximation on mathematics achievement is fully mediated by symbolic approximation (Complete Mediation model).

Model fit was assessed according to commonly accepted cutoff criteria (Hu \& Bentler, 2009; Schermelleh-Engel \& Moosbrugger, 2003). These include the Comparative Fit Index (CFI) and the Tucker-Lewis Index (TLI) being close to .95 or greater, the Root Mean Square Error of Approximation (RMSEA) and the Standardized Root Mean Residual (SRMR) being close to .05 or smaller. The $\chi^{2}$ fit statistic test was not directly evaluated due to its known drawbacks with large sample sizes. Instead, incremental fit was assessed with the $\chi^{2} / \mathrm{df}$ fit value (Schermelleh-Engel \& Moosbrugger, 2003), in which values close to 2 or smaller indicate good model fit.

The aforementioned absolute goodness of fit measures were used to compare the two measurement models (CFA1 vs CFA2). In the case of the SEM models, the full model (Partial Mediation) was compared with the fit of the Direct Effects and the Complete Mediation model by conducting chi-square difference tests (Satorra \& Bentler, 2001). To facilitate model comparison, goodness-of-fit values, fit indexes and their corresponding acceptable and good fit criteria are summarized in Table 3. 


\subsection{Results}

\subsubsection{Preliminary Analyses}

To examine whether the targeted prediction pattern of math achievement was influenced by the school factor, we calculated intra class correlations (ICCs) for all observed predictor variables. Our results showed that in 6 out of the 8 observed variables, the ICC results were very small and thus negligible: nonsymbolic approximate addition: 0.005 , nonsymbolic approximate comparison: 0.010 , symbolic approximate addition: 0.021 , symbolic approximate comparison: 0.089 , word recall forward: 0.034 , word recall backwards: 0.047. The Dot Matrix demonstrated a medium ICC $(0.105)$ and only performance in the Odd One Out task demonstrated a high ICC (0.264). The Odd One Out task comprises only one of the four measures used to assess the WM construct. Additionally, there was no plausible reason to suppose that the school factor would influence performance on this task. Therefore, for model parsimony, the school factor was not included in our models.

To establish the validity of our nonsymbolic and symbolic approximate tasks, we examined whether children scored above chance level and showed the expected ratio effect. $T$-test analyses showed that participants performed above chance level (50\%) in all four approximation tasks: nonsymbolic addition $(M=63.04 \%, t(438)=25.13, p<.001)$, nonsymbolic comparison $(M=64.32 \%, t(438)=22.50, p<.001)$, symbolic addition $(M=$ $56.53 \%, t(438)=11.62, p<.001)$, and symbolic comparison $(M=60.02 \%, t(438)=13.79, p$ $<.001)$. Supplementary analyses indicated that children solved the tasks without resorting to any guessing or comparison strategies alternative to approximate computations (Appendix, Table A2). Furthermore, they did not base their responses on the physical features of the dots in the case of the nonsymbolic versions (Appendix, Table A3). 
The characteristic ratio effect was evident in children's performance in the nonsymbolic approximate comparison, $F(2,880)=88.49, p<.001$, and addition task $F(1.96$, $862.47)=113.71, p<.001$ (Figure 2). We also observed it for the symbolic approximate comparison task, $F(1.94,851.18)=13.69, p<.001$, but not for the symbolic approximate addition task, $F(1.96,860.16)=1.56, p=.211($ Figure 2$)$.

- Insert Figure 2 -

\subsubsection{Descriptive Statistics}

Descriptive statistics of the administered measures are presented in Table 1.

Probability distribution descriptors indicated that all measures were within the acceptable limits of skewness less than 3 and kurtosis less than 4 (Kline, 2011). Table 2 entails the correlations amongst the observed variables.

- Insert Table 1 -

- Insert Table 2 -

\subsubsection{Measurement Models}

CFA analyses were conducted to determine the latent factor structure of the skills assessed. The CFA1 (with a single system of approximation) demonstrated a poor fit to the data and was rejected on the basis of the absolute goodness of fit indices, CFI, TLI and 
RMSEA (Table 3). In contrast, the CFA2, where, as hypothesized, nonsymbolic and symbolic approximation comprised two separate abilities, demonstrated a good fit to the data, $\chi^{2} / d f=$ $1.99, C F I=.969, T L I=.951, R M S E A=.047, S R M R=.033$. As shown in Figure 3 , in the

CFA2 all observed variables loaded on the respective latent variables and all factor loadings were significant $(p \leq .001)$, with moderate to high effects ranging from .40 to .84 .

- Insert Figure 3 -

\subsubsection{SEM analyses}

With the CFA2 as the best fitting measurement model, we moved to examine the structural associations of the latent variables and the hypotheses regarding the role of nonsymbolic and symbolic approximation in kindergarteners' math achievement. Three SEM analyses were conducted. First, we tested the Partial Mediation model, which assumes that symbolic approximation partially mediates the effect of nonsymbolic approximation on children's math achievement. This model demonstrated a good fit to our data, $\chi^{2} / d f=1.99$, $C F I=.969, T L I=.951, R M S E A=.047, S R M R=.033$. All structural path coefficients were significant ( $p \leq .001 ; \beta$ s ranging from .28 to .70 ), with the exception of the non-significant path between nonsymbolic approximation and math achievement $(\beta=.13, p=.071)$.

Next, the Direct Effects model was tested, where direct effects of nonsymbolic approximation and symbolic approximation on math achievement were specified. This model demonstrated a somewhat poorer fit, $\chi^{2} / d f=2.47, C F I=.952, T L I=.927, R M S E A=.058$, $S R M R=.046$. Again, all structural paths were significant $(p \leq .001)$ with $\beta$ s ranging from .25 
to .74 apart from the direct path between nonsymbolic approximation and math achievement $(\beta=.11, p=.115)$

In the Complete Mediation model symbolic approximation skills completely mediated the effect of nonsymbolic approximation skills on math achievement (Figure 4). This model demonstrated good fit, $\chi^{2} / d f=2.02, C F I=.966, T L I=.950, R M S E A=.048, S R M R=.034$. All structural paths were statistically significant in the hypothesized directions $(p \leq .001)$.

- Insert Figure 4 -

Chi-square difference tests for nested models were conducted to compare the Partial Mediation model (full model) with the Direct Effects and the Complete Mediation model. The Partial Mediation model fit the data significantly better than the Direct Effect model, $\Delta \chi^{2}(1)=$ 74.24, $p=.001$, but not compared to the Complete Mediation model, $\Delta \chi^{2}(1)=3.07, p=.08$. These results suggested that, the more parsimonious, Complete Mediation model was the final model. Thus, symbolic approximation skills were shown to completely mediate the effect of nonsymbolic approximation skills on math achievement when controlling for WM effects. As shown in Figure 4, the expected associations between WM capacity, approximation skills and math achievement were demonstrated. Individual differences in WM capacity explained performance variation in both nonsymbolic and symbolic kindergarten approximation. Furthermore, WM capacity highly predicted individual differences in math achievement beyond the effect of approximation. Path coefficients of structural parameters in this final model (Figure 4), ranged from .30 to .74 representing medium to large effects (Cohen, 1992) 
in the expected directions. With this model structure, $87.2 \%(S E=.064, p<.001)$ of the variance of kindergarteners' math achievement was explained.

- Insert Table 3 -

\subsection{Discussion}

This large-scale study took an integrative look into the associations between approximation, WM and kindergarteners' math skills. Results confirmed the expected factorial structure of the integrative theoretical model. Approximation was comprised of two distinct cognitive systems: nonsymbolic and symbolic approximation. Both systems correlated with kindergarteners' counting and exact addition skills beyond the effect of WM capacity. We showed that the predictive relation between nonsymbolic approximation and math achievement is indirect. It was completely mediated by symbolic approximation. As expected, WM gave rise to individual differences in both nonsymbolic and symbolic approximation skills. Lastly, as expected, individual differences in WM highly predicted individual differences in math achievement beyond the effects of approximation skills. The final model (Complete Mediation), which entailed these integrative effects, demonstrated a very good fit to the data and explained $87.2 \%$ of the variance of kindergarteners' counting and exact addition skills. In the following section, we discuss our findings more elaborately and propose future research directions.

Our preliminary analyses replicated findings regarding kindergarteners' nonsymbolic and symbolic approximation skills. Children performed above chance level in all approximation tasks (addition and comparison) without resorting to any guessing or 
comparison strategies alternative to approximation (Barth et al., 2006; Gilmore et al., 2010; Xenidou-Dervou et al., in press). Moreover, we observed the characteristic ratio effect for both nonsymbolic approximate tasks (Barth et al., 2006; Gilmore et al., 2010; XenidouDervou et al., in press). The ratio effect was also evident for the direct comparison symbolic condition (Gilmore et al., 2007) but not for the condition that asked for the symbolic approximate addition of two quantities. While all numerical tasks had good reliability and loaded highly on their corresponding latent factors, the differences between the current data and those of Gilmore et al. (2007) may be due to task administration or sample characteristic differences.

Results regarding the measurement models highlighted the distinct but also integrative relation between nonsymbolic and symbolic representations of quantity. The ability of representing nonsymbolic numerosities was distinct to that of representing symbolic numerals (Holloway \& Ansari, 2009). The measurement model with the two approximation abilities (nonsymbolic and symbolic) demonstrated the best fit to our data. In this model, we observed the expected correlations amongst the four different latent constructs (WM, nonsymbolic and symbolic approximation, and math achievement). WM correlated with mathematical achievement (DeStefano \& LeFevre, 2004), as well as nonsymbolic (Xenidou-Dervou et al., in press) and symbolic approximate skills (Caviola et al., 2012). Very few studies have made use of multiple approximation measures. Using both addition and comparison approximation measures, we were able to demonstrate the interrelationship of the two approximation skills in the acquisition process of early mathematical knowledge. Nonsymbolic approximation correlated with symbolic approximation (Gilmore et al., 2007; 2010; Libertus, et al., 2011) and both correlated with mathematical achievement in kindergarten (Gilmore et al., 2010; Halberda, Mazzocco, \& Feigenson, 2008; Libertus, et al., 2011). The latter finding suggests that both skills are possibly operative in the early steps of symbolic arithmetic learning. 
But what differential roles do nonsymbolic and symbolic approximation play in the early process of math learning? This topic has been an ongoing issue of debate in the literature (e.g. Gilmore et al., 2010; Mazzocco et al., 2011; Noël \& Rouselle, 2011; Sasanguie et al., 2011). Consistent with earlier assumptions (Gilmore et al., 2007; 2010; Mundy \& Gilmore, 2009), our final model showed that individual differences in nonsymbolic approximation predicted individual differences in symbolic approximation. In other words, symbolic approximate numerical representation skills were indicated to map onto preexisting nonsymbolic representations. Moreover, both nonsymbolic and symbolic approximation skills correlated with kindergarteners' math learning beyond the effect of WM. The predictive path of the first, however, was an indirect one. The role of nonsymbolic approximation was completely mediated by symbolic approximation.

This finding could explain certain incongruent assumptions within the literature. Research focusing solely on the role of nonsymbolic approximate magnitude representation has shown it to account for individuals' differences in early math performance (e.g. Gilmore et al., 2010; Libertus et al., 2011; Mazzocco et al., 2011). This lead to the assumption that the ANS is an important stepping-stone for the development of mathematical skills. On the other hand, studies targeting symbolic approximate magnitude representation (e.g. De Smedt, Verschaffel, \& Ghesquière, 2009b) or addressing it in conjunction with nonsymbolic mental representation (e.g. De Smedt \& Gilmore, 2010; Holloway \& Ansari, 2009; Sasanguie, et al., 2011) highlighted the first as the best early precursor of math achievement. Our findings suggest that the two abilities should not be viewed as two competing predictors. It is not one or the other per se that is the best predictor. Both nonsymbolic and symbolic approximate representation skills correlated with kindergarten math achievement and it was these skills' mediating relationship that best explained kindergarteners' individual differences. We showed 
that in kindergarten, nonsymbolic approximation predicts counting and exact symbolic addition indirectly.

The aforementioned view contrasts the assumption that nonsymbolic approximate magnitude skills do not play an important role in early arithmetic (e.g. LeCorre \& Carrey, 2007; Noël \& Rouselle, 2011). Of course, as highlighted by Noël and Rouselle, (2011), the incongruence in conclusions regarding the role of nonsymbolic skills is also due to differences in the age of children tested in the various studies. In their critical overview of research in atypical mathematics development, Noël and Rouselle, (2011) highlight that the effect of nonsymbolic numerical skills becomes pronounced in later ages. The authors propose a bidirectional model of effects between nonsymbolic approximate number representation and exact symbolic representation. ANS acuity increases with age (Halberda \& Feigenson, 2008) and as educated humans we develop the ability for higher-order mathematics utilizing symbolic stimuli. Based on our findings, we suggest that future research should focus on the integrative associations of nonsymbolic and symbolic approximation and WM. How does the pattern of predictive relations change with age?

Consistent with previous research (Bull et al., 2008; De Smedt et al., 2009a; Passolungi et al., 2007; Swanson \& Kim, 2007), WM was confirmed to be a significant independent predictor of individual differences in kindergarten math achievement. Our results extend previous findings by showing that WM significantly predicts symbolic arithmetic learning beyond domain-specific skills, such as approximation skills. Additionally, individual differences in WM gave rise to individual differences in both nonsymbolic and symbolic approximation (Caviola et al., 2012; Xenidou-Dervou et al., in press). Consequently, WM appears as an independent predictor that sets the foundations from which approximation abilities are influenced by in kindergarten. Future research should look into the developmental trajectories of these relationships and the role of the different WM components. It is known 
that the role of WM capacity used in given tasks changes with age as children eventually start employing different, more sophisticated problem solving strategies (e.g., Imbo \& Vandierendonck, 2007; McKenzie, Bull \& Gray, 2003; Rasmussen \& Bisanz, 2005).

Limitations of this study should be addressed in future research. Because we only tested the children on one PL measure and one VSSP measure, it was not optimal to address the different WM components as separate latent constructs. Future studies could enhance the integrative model by including the roles of the separate WM components and their effects on approximation and early math skills. Furthermore, as the present study was based on data collected at one time-point, future research should address the integrative model's assumptions longitudinally.

In summary, we examined the cognitive architectural system that explains individual performance variation in kindergarten mathematics achievement by taking an integrative look into the associations between nonsymbolic approximation, symbolic approximation and WM capacity. Our final model explained a very high percentage of kindergarteners' differences in learning counting and exact addition. It is important for future research to further elaborate on the relationship between approximation skills and WM as interactive building blocks with the scope of facilitating early math proficiency and deficiency prediction. Recognizing not only the predictors but also their interrelations will draw a clearer picture on how educational practice can help children build a solid cognitive foundation for learning math. 
References

Akaike, H. (1974). A new look at the statistical model identification. IEEE Transactions on Automatic Control, 19, 716-723.

Alloway, T. P. (2007). Automated working memory assessment. London: Psychological Corporation.

Alloway, T. P., Gathercole, S. E., \& Pickering, S. J. (2006). Verbal and visuospatial shortterm and working memory in children: are they separable? Child Development, 77, 1698-1716. doi:10.1111/j.1467-8624.2006.00968.x

Ashcraft, M. H., \& Fierman, B. A. (1982). Mental addition in $3^{\text {rd }}, 4^{\text {th }}$, and $6^{\text {th }}$ graders. Journal of Experimental Child Psychology, 33, 216-234.

Baddeley, A. D. (1986). Working memory. New York: Clarendon.

Baddeley, A. (2003). Working memory: Looking back and looking forward. Nature Reviews Neuroscience, 4, 829-839. doi:10.1016/S0021-9924(03)00019-4

Barth, H., Beckmann, L., \& Spelke, E. S. (2008). Nonsymbolic, approximate arithmetic in children: abstract addition prior to instruction. Developmental Psychology, 44, 14661477. doi: $10.1037 / \mathrm{a} 0013046$

Barth, H., La Mont, K., Lipton, J., \& Spelke, E. S. (2005). Abstract number and arithmetic in preschool children. Proceedings of the National Academy of Sciences of the United States of America, 102, 14116-14121. doi: 10.1073/pnas.0505512102

Barth, H., La Mont, K., Lipton, J., Dehaene, S., Kanwisher, N., \& Spelke, E. (2006). Nonsymbolic arithmetic in adults and young children. Cognition, 98, 199-222. doi: 10.1016/j.cognition.2004.09.011 
Brannon, E. M., Jordan, K. E., \& Jones, S. M. (2010). Behavioral signatures of numerical discrimination. In M. L. Platt \& A.A. Ghazanfar (Eds.), Primate neuroethology (pp. 144-159). Oxford: Oxford University Press.

Bull, R., Espy, K. A., \& Wiebe, S. A. (2008). Short-term memory, working memory and executive functioning in preschoolers: Longitudinal predictors of mathematical achievement at age 7 years. Developmental Neuropsychology, 33, 205-228.doi: $10.1080 / 87565640801982312$

Caviola, S., Mammarella, I. C., Cornoldi, C., \& Lucangeli, D. (2012). The involvement of working memory in children's exact and approximate mental addition. Journal of Experimental Child Psychology, 112, 141-160. doi: 10.1016/j.jecp.2012.02.005

Cohen, J. (1992). A power primer. Psychological Bulletin, 112, 155-159.

Dehaene, S. (2001). Précis of the number sense. Mind \& Language, 16, 16-36.

Dehaene, S. (2011). The Number Sense: How the Mind Creates Mathematics, Revised and Updated Edition. New York, NY: Oxford University Press, USA.

De Smedt, B., \& Gilmore, C. K. (2011). Defective number module or impaired access? Numerical magnitude processing in first graders with mathematical difficulties. Journal of Experimental Child Psychology, 108, 278-292. doi:10.1016/j.jecp.2010.09.003

De Smedt, B., Janssen, R., Bouwens, K., Verschaffel, L., Boets, B., \& Ghesquière, P. (2009a). Working memory and individual differences in mathematics achievement: A longitudinal study from first grade to second grade. Journal of Experimental Child Psychology, 103, 186-201. doi:10.1016/j.jecp.2009.01.004 
De Smedt, B., Verschaffel, L., \& Ghesquière, P. (2009b). The predictive value of numerical magnitude comparison for individual differences in mathematics achievement. Journal of Experimental Child Psychology, 103, 469-479. doi:10.1016/j.jecp.2009.01.010

DeStefano, R., \& LeFevre, J. (2004). The role of working memory in mental arithmetic. European Journal of Cognitive Psychology, 16, 353-386. doi:10.1080/09541440244000328

Feigenson, L., Dehaene, S., \& Spelke, E. (2004). Core systems of number. Trends in Cognitive Science, 8, 307-314. doi:10.1016/j.tics.2004.05.002

Geary, D. C. (2011). Consequences, characteristics, and causes of mathematical learning dissabilities and persistent low achievement in mathematics. Journal of Developmental \& Behavioral Pediatrics, 32, 251-263. doi:10.1097/DBP.0b013e318209edef

Geary, D.C., Hoard, M. K., Byrd-Craven, J., \& DeSoto, M. C. (2004). Strategy choices in simple and complex addition: Contributions of working memory and counting knowledge for children with mathematical disability. Journal of Experimental Child Psychology, 88, 121-151. doi:10.1016/j.jecp.2004.03.002

Gilmore, C. K., McCarthy, S. E., \& Spelke, E. S. (2007). Symbolic arithmetic knowledge without instruction. Nature, 447, 589-591. doi:10.1038/nature05850

Gilmore, C. K., McCarthy, S. E., \& Spelke, E. S. (2010). Non-symbolic arithmetic abilities and mathematics achievement in the first year of formal schooling. Cognition, 115, 394406. doi:10.1016/j.cognition.2010.02.002

Gullick, M. M., Sprute, L. A., \& Temple, E. (2011). Individual differences in working memory, nonverbal IQ, and mathematics achievement and brain mechanisms associated 
with symbolic and nonsymbolic number processing. Learning and Individual Differences, 21, 644-654. doi:10.1016/j.lindif.2010.10.003

Halberda, J., \& Feigenson, L. (2008). Developmental change in the acuity of the "Number Sense": The approximate number system in 3-, 4-, 5-, and 6-year-olds and adults. Developmental Psychology, 44, 1457-1465. doi:10.1037/a0012682

Halberda, J., Mazzocco, M. M. M., \& Feigenson, L. (2008). Individual differences in nonverbal number acuity correlate with maths achievement. Nature, 445, 665-669. doi:10.1038/nature07246

Hamann, M. S., \& Ashcraft, M. H. (1985). Simple and complex mental addition across development. Journal of Experimental Child Psychology, 40, 49-72. doi:10.1016/0022-0965(85)90065-7

Holloway, I. D., \& Ansari, D. (2009). Mapping magnitudes onto symbols. The numerical distance effect and individual differences in children's mathematics achievement. Journal of Experimental Child Psychology, 103, 17-29. doi:10.1016/j.jecp.2008.04.001

Hoyle, R. H. (1995). The structural equation modeling approach: Basic concepts and fundamental issues. In: Hoyle, Rick H. (Ed.). Structural equation modeling: Concepts, issues, and applications (pp. 1-15). Thousand Oaks, CA: SAGE Publications.

Hu, L. \& Bentler, P. M. (2009). Cutoff criteria for fit indexes in covariance structure analysis: conventional criteria versus new alternatives. Structural Equation Modeling: A Multidisciplinary Journal, 6, 1-55. 
Imbo, I., \& Vandierendonck, A. (2007). The role of phonological and executive working memory resources in simple arithmetic strategies. European Journal of Cognitive Psychology, 19, 910-933. doi:10.1080/09541440601051571

Izard, V., \& Dehaene, S. (2008). Calibrating the mental number line. Cognition, 106, 12211247. doi:10.1016/j.cognition.2007.06.004

Jenks, K. M., De Moor, J., \& Van Lieshout, E. C. D. M. (2009). Arithmetic difficulties in children with cerebral palsy are related to executive function and working memory. Journal of Child Psychology and Psychiatry, 50, 824-833. doi:10.1111/j.14697610.2008.02031.x

Kline, R. B. (2011). Principles and practice of structural equation modeling ( ${ }^{\text {rd }}$ ed.). NY: Guilford.

Le Corre, M., \& Carey, S. (2007). One, two, three, four, nothing more: an investigation of the conceptual sources of the verbal counting principles. Cognition, 105, 395-438. doi:10.1016/j.cognition.2006.10.005

LeFevre, J., DeStefano, D., Coleman, B., \& Shanahan, T. (2005). Mathematical cognition and working memory. In J. I. D. Campbell (Ed.), The handbook of mathematical cognition (pp. 361-378). New York: Psychology Press.

Libertus, M. E., Feigenson, L., \& Halberda, J. (2011). Preschool acuity of the approximate number system correlates with school math ability. Developmental Science, 14, 12921300. doi:10.1111/j.1467-7687.2011.01080.x

Mazzocco, M. M. M., Feigenson, L., \& Halberda, J. (2011). Preschooler's precision of the approximate number system predicts later school mathematics performance. PlosOne 6 (9). doi:10.1371/journal.pone.0023749 
Messer, M. H., Leseman, P. P. M., Boom, J. Mayo, A. Y. (2010). Phonotactic probability effect in nonword recall and its relationship with vocabulary in monolingual and bilingual preschoolers. Journal of Experimental Child Psychology, 105, 306-323. doi:10.1016/j.jecp.2009.12.006

McKenzie, B., Bull, R., \& Gray, C. (2003). The effects of phonological and visual-spatial interference on children's arithmetical performance. Educational and Child Psychology, 20, 93-108.

McNeil, N. M., Wagner Fuhs, M., Keultjes, M. C., \& Gibson, M. H. (2011). Influences of problem format and SES on preschoolers' understanding of approximate addition. Cognitive Development, 26, 57-71. doi:10.1016/j.cogdev.2010.08.010

Mundy, E., \& Gilmore, C. K. (2009). Children's mapping between symbolic and nonsymbolic representations of number. Journal of Experimental Child Psychology, 103, 490-502. doi:10.1016/j.jecp.2009.02.003

Muthén, L. K., \& Muthén, B. O. (1998). Mplus user's guide. Muthén and Muthén, Los Angeles, CA.

Noël, M. P. (2009). Counting on working memory when learning to count and add: a preschool study. Developmental Psychology, 45, 1630-1643. doi:10.1037/a0016224

Noël, M. P., \& Rouselle, L. (2011). Developmental changes in the profiles of dyscalculia: an explanation based on a double exact-and-approximate number representation model. Frontiers in Human Neuroscience, 5: 165. doi:10.3389/fnhum.2011.00165

Passolunghi, M. C., Vercelloni, B., \& Schadee, H. (2007). The precursors of mathematics learning: Working memory, phonological ability, and numerical competence. Cognitive Development, 22, 165-184. doi:10.1016/j.cogdev.2006.09.001 
Piazza, M. (2010). Neurocognitive start-up tools for symbolic number representations. Trends in Cognitive Science, 14, 542-551. doi:10.1016/j.tics.2010.09.008

Piazza, M., Facoetti, A.,Trussardi, A. N., Berteletti, I.,Conte, S., Lucangeli,D., Dehaene, S., and Zorzi, M. (2010). Developmental trajectory of number acuity reveals a severe impairment in developmental dyscalculia. Cognition 116, 33-41. doi:10.1016/j.cognition.2010.03.012

Raghubar, K. P., Barnes, M. A., \& Hecht, S. A. (2010). Working memory and mathematics: A review of developmental, individual difference and cognitive approaches. Learning and Individual Differences, 20, 110-122. doi: 10.1016/j.lindif.2009.10.005

Rasmussen, C., \& Bisanz, J. (2005). Representation and working memory in early arithmetic. Journal of Experimental Child Psychology, 91, 137-157. doi: 10.1016/j.jecp.2005.01.004

Repovš, G., \& Baddeley, A. (2006). The multi-component model of working memory: explorations in experimental cognitive psychology. Neuroscience, 139, 5-21. doi: 10.1016/j.neuroscience.2005.12.061

Sasanguie, D., De Smedt, B., Defever, E., \& Reynvoet, B. (2011). Association between basic numerical abilities and mathematics achievement. British Journal of Developmental Psychology, 30, 344-357. doi:10.1111/j.2044-835X.2011.02048.x.

Simmons, F. R., Willis, C., \& Adams, A. (2012). Different components of working memory have different relationships with different mathematical skills. Journal of Experimental Child Psychology, 111, 139-155. doi:10.1016/j.jecp.2011.08.011

Schwarz, G. (1978). Estimating the dimension of a model. The Annals of Statistics, 6, 461466. 
Schermelleh-Engel, K., \& Moosbrugger, H. (2003). Evaluating the fit of structural equation models: tests of significance and descriptive goodness-of-fit measures. Methods of Psychological Research Online, 8, 23-74.

Swanson, L., \& Kim, K. (2007). Working memory, short-term memory, and naming speed as predictors of children's mathematical performance. Intelligence, 35, 151-168. doi:10.1016/j.intell.2006.07.001

Toll, S. W. M., Van der Ven, S. H. G., Kroesbergen, E. H., \& Van Luit, J. E. H. (2011). Executive functions as predictors of learning disabilities. Journal of Learning Disabilities, 44, 521-532. doi:10.1177/0022219410387302

Van Luit, J. E. H., \& Van de Rijt, B. A. M. (2009). Utrechtse Getalbegrip Toets-Revised [Early Numeracy Test-Revised]. Doetinchem: Graviant.

Xenidou-Dervou, I., van Lieshout, E. C. D. M. and van der Schoot, M. (in press), Working Memory in Nonsymbolic Approximate Arithmetic Processing: A Dual-Task Study With Preschoolers. Cognitive Science. doi:10.1111/cogs.12053. 


\section{Acknowledgements}

The authors would like to thank all children, parents, teachers and schools that participated in this research and the entire $\mathrm{XXXXX}^{2}$ team. We would also like to thank $\mathrm{XXX}$ for his invaluable help in developing the approximation tasks and XXXX, XXXX and XXXX for their advice on the SEM analyses. This research was conducted as part of a broader XXXX funded project.

\footnotetext{
${ }^{2}$ Information omitted for blind review
} 


\section{Appendix}

Our (non)symbolic approximate tasks were designed on the basis of the nonsymbolic approximate addition task used in Xenidou-Dervou et al. (in press). In order to further verify the validity of the newly developed tasks, it was important to examine whether kindergarteners in our study made use of any alternative response strategies, such as for example pressing systematically only the blue button. Furthermore, we examined whether the children's responses in the nonsymbolic conditions (addition and comparison) were based on continuous quantity characteristics of the stimuli, in other words, the physical features of the dots. As in previous studies (Barth et al., 2006; Gilmore et al., 2010; Xenidou-Dervou et al., in press) the effect of these biases were controlled for in the trial construction level of these tasks.

\section{Alternative Strategy use}

Table A1 adduces the 24 testing trials that were presented during each of the four approximate tasks: nonsymbolic approximate addition, nonsymbolic approximate comparison, symbolic approximate addition and symbolic approximate comparison. In this table, trials are listed with a 1 or -1 under certain systematic response strategies. A trial listed as 1 signified that the use of the corresponding strategy would lead to a correct response on this specific trial. A trial listed as -1 signified that the use of this strategy would lead to an incorrect response. Performance on trials instigating the use of a possible systematic strategy were compared to chance level performance (50\%). Table A2 summarizes these results.

For the nonsymbolic approximate addition task, we examined the use of five different possible systematic response strategies. If children had shown a response bias such as pressing always the blue or the red button (Blue and Red systematic strategies), then they would have performed at chance level. Results showed that such strategies were not used; 
Table A1. Testing Trials of the Nonsymbolic and Symbolic Approximate Tasks from Xenidou-Dervou et al. (in press)

\begin{tabular}{|c|c|c|c|c|c|c|c|c|c|c|c|c|}
\hline & \multirow{2}{*}{ Ratio } & \multirow{2}{*}{ First blue array (B1) } & \multirow{2}{*}{ Second blue array (B2) } & \multirow{2}{*}{ Sum of blue } & \multirow{2}{*}{ Red array (R) } & \multirow{2}{*}{ Correct Response } & \multicolumn{5}{|c|}{ Systematic strategies $^{a}$} & \multirow{2}{*}{$\begin{array}{c}\text { Continuous } \\
\text { Quantity }^{c}\end{array}$} \\
\hline & & & & & & & Near/far & Blue & Red & B2 vs $R$ & B1 vs $\mathbf{R}$ & \\
\hline 1 & $4: 7$ & 6 & 6 & 12 & 21 & Red & Far (0.29) & $-1^{\mathrm{b}}$ & 1 & 1 & 1 & $\mathrm{~B}$ \\
\hline 2 & $4: 7$ & 8 & 12 & 20 & 35 & Red & Medium (0.34) & -1 & 1 & 1 & 1 & $\mathrm{~A}$ \\
\hline 3 & $4: 7$ & 15 & 13 & 28 & 49 & Red & $\operatorname{Far}(0.31)$ & -1 & 1 & 1 & 1 & $\mathrm{~B}$ \\
\hline 4 & $4: 7$ & 16 & 20 & 36 & 63 & Red & $\operatorname{Far}(0.32)$ & -1 & 1 & 1 & 1 & A \\
\hline 5 & $7: 4$ & 20 & 8 & 28 & 16 & Sum Blue & Near $(0.80)$ & 1 & -1 & -1 & 0 & $\mathrm{~B}$ \\
\hline 6 & $7: 4$ & 30 & 12 & 42 & 24 & Sum Blue & Near $(0.80)$ & 1 & -1 & -1 & 0 & A \\
\hline 7 & $7: 4$ & 6 & 50 & 56 & 32 & Sum Blue & Medium (0.64) & 1 & -1 & 1 & -1 & $\mathrm{~B}$ \\
\hline 8 & $7: 4$ & 7 & 63 & 70 & 40 & Sum Blue & Medium (0.63) & 1 & -1 & 1 & -1 & A \\
\hline 9 & $4: 6$ & 8 & 8 & 16 & 24 & Red & Far $(0.33)$ & -1 & 1 & 1 & 1 & $\mathrm{~B}$ \\
\hline 10 & $4: 6$ & 16 & 8 & 24 & 36 & Red & Medium (0.44) & -1 & 1 & 1 & 1 & A \\
\hline 11 & $4: 6$ & 12 & 20 & 32 & 48 & Red & Medium (0.42) & -1 & 1 & 1 & 1 & B \\
\hline 12 & $4: 6$ & 20 & 20 & 40 & 60 & Red & Far (0.33) & -1 & 1 & 1 & 1 & A \\
\hline 13 & $6: 4$ & 11 & 7 & 18 & 12 & Sum Blue & Near $(0.92)$ & 1 & -1 & -1 & 0 & $\mathrm{~B}$ \\
\hline 14 & $6: 4$ & 33 & 7 & 42 & 28 & Sum Blue & Near $(0.85)$ & 1 & -1 & -1 & 0 & A \\
\hline 15 & $6: 4$ & 25 & 35 & 60 & 40 & Sum Blue & Near $(0.88)$ & 1 & -1 & 0 & -1 & B \\
\hline 16 & $6: 4$ & 10 & 26 & 36 & 24 & Sum Blue & Near $(0.92)$ & 1 & -1 & 0 & -1 & A \\
\hline 18 & $4: 5$ & 12 & 12 & 24 & 30 & Red & Medium $(0.40)$ & -1 & 1 & 1 & 1 & A \\
\hline 19 & $4: 5$ & 24 & 8 & 32 & 40 & Red & Medium (0.60) & -1 & 1 & 1 & 1 & B \\
\hline 20 & $4: 5$ & 34 & 6 & 40 & 50 & Red & Near $(0.68)$ & -1 & 1 & 1 & 1 & A \\
\hline 21 & $5: 4$ & 6 & 14 & 20 & 16 & Sum Blue & Near $(0.88)$ & 1 & -1 & 0 & -1 & B \\
\hline 22 & $5: 4$ & 15 & 50 & 65 & 52 & Sum Blue & Near $(0.96)$ & 1 & -1 & 0 & -1 & A \\
\hline 23 & $5: 4$ & 32 & 8 & 40 & 32 & Sum Blue & Near (1.00) & 1 & -1 & -1 & 0 & B \\
\hline 24 & $5: 4$ & 40 & 10 & 50 & 40 & Sum Blue & Near (1.00) & 1 & -1 & -1 & 0 & A \\
\hline
\end{tabular}

${ }^{a}$ These columns present information for the given trials with regard to the usage of possible systematic response strategies: Near/far $=$ response based on the ratio distance between the larger blue addend and the red; Blue = only the blue response is chosen; Red = only the red response is chosen; B2 vs Rstr = only the second blue addend is compared; B1vsRstr only the first blue addend is compared,

${ }^{\mathrm{b}} 1=$ predicts correct answer for that trial, -1 : predicts a false answer for that trial, $0=$ does not provide a clear prediction

${ }^{\mathrm{c}}$ Continuous quantity conditions: $\mathrm{A}=\mathrm{dot}$ size, total dot surface area, total dot contour length and density positively correlated with number while array size negatively correlated with number;

$\mathrm{B}=$ dot size, total dot surface area, total dot contour length and density negatively correlated with number while array size positively correlated with number 


\section{KINDERGARTEN MATH ACHIEVEMENT}

kindergarteners performed significantly above chance in both cases. In the addition tasks, where two blue quantities were presented, there was the possibility that children based their answer only on the relative quantitative distance of the larger of the blue addends and the red addend (Near/far strategy). If this distance was small (near) than the child could chose in favor of the blue addends, if it was large (red), then he/she would opt for the red array being the correct answer. Therefore, trials with this distance level being of medium size were compared to chance level performance. Our findings did not indicate the use of this strategy. Another strategy regarded the possibility of children preferring to compare only one of the two blue addends with the red, instead of mentally adding the two blue and then comparing them to the target. This was not the case; children did not show a preference for comparing the first blue array with the red (B1vsR strategy) or the second blue array with the red (B2vsR strategy).

Table A2

Assessing the Use of Systematic Response Preferences in our Nonsymbolic and Symbolic Approximate tasks.

\begin{tabular}{|c|c|c|c|c|c|}
\hline Task & Strategy & $\begin{array}{l}\text { Number of } \\
\text { Trials }\end{array}$ & $\begin{array}{c}\text { Mean Accuracy } \\
\%\end{array}$ & $p$ - value & $\begin{array}{c}>\text { Chance } \\
(50 \%)\end{array}$ \\
\hline \multirow{5}{*}{$\begin{array}{l}\text { Nonsymbolic approximate } \\
\text { addition }\end{array}$} & Blue & 12 & 59.56 & .000 & yes \\
\hline & Red & 12 & 66.42 & .000 & yes \\
\hline & Near/Far & 8 & 61.36 & .000 & yes \\
\hline & B1vsR & 6 & 69.46 & .000 & yes \\
\hline & B2vsR & 6 & 63.38 & .000 & yes \\
\hline \multirow[t]{5}{*}{ Symbolic approximate addition } & Blue & 12 & 57.39 & .000 & yes \\
\hline & Red & 12 & 55.62 & .000 & yes \\
\hline & Near/Far & 8 & 56.93 & .000 & yes \\
\hline & B1vsR & 6 & 58.71 & .000 & yes \\
\hline & $\mathrm{B} 2 \mathrm{vsR}$ & 6 & 52.54 & .064 & no \\
\hline \multirow{2}{*}{$\begin{array}{l}\text { Nonsymbolic approximate } \\
\text { comparison }\end{array}$} & Blue & 12 & 63.30 & .000 & yes \\
\hline & Red & 12 & 65.27 & .000 & yes \\
\hline \multirow{2}{*}{$\begin{array}{l}\text { Symbolic approximate } \\
\text { comparison }\end{array}$} & Blue & 12 & 65.42 & .000 & yes \\
\hline & Red & 12 & 54.58 & .000 & yes \\
\hline
\end{tabular}

The possible use of the same systematic strategies was also examined for the case of the symbolic approximate addition task. Similarly, children performed above chance level in 


\section{KINDERGARTEN MATH ACHIEVEMENT}

all response bias (Blue and Red strategy) and non-addition strategies (Near/far, B1vsR) apart from the case of the B2vsR strategy (see Table A2). In this case, their performance was not significantly above chance level; it was also, though, not significantly below chance level indicating that this non-addition strategy was also not employed.

In the nonsymbolic and symbolic approximate comparison tasks there was only one blue quantity and one red quantity. Therefore, only the response bias for opting to choose either the red or the blue button was examined. As seen in Table A2 in both tasks no such systematic response preference was utilized.

\section{Continuous quantity strategies}

In nonsymbolic tasks it is possible for children to rely their responses on the features of the dots such as their size or the total size of a dot-array. Similar to the tasks used by Gilmore and colleagues (2010), in our nonsymbolic tasks we controlled for physical feature related variables by presenting the stimuli in two distinct conditions. In condition A, dot size, total dot surface area, total dot contour length and density of the dots was positively correlated with the respective numerosity of the stimulus. Condition B had the reverse relations. As shown in Table A3, in the approximate addition task children performed above

\section{Table A3}

Continuous Quantity Strategy Analyses

\begin{tabular}{cccccc}
\hline Task & $\begin{array}{c}\text { Continuous } \\
\text { Quantity }^{\text {cus }}\end{array}$ & $\begin{array}{c}\text { Number of } \\
\text { Trials }\end{array}$ & $\begin{array}{c}\text { Mean } \\
\text { Accuracy } \%\end{array}$ & $\boldsymbol{p}$ - value & $\begin{array}{c}\text { Chance } \\
\mathbf{( 5 0 \% )}\end{array}$ \\
\hline Nonsymbolic approximate & $\mathrm{A}$ & 12 & 52.34 & .027 & yes \\
addition & $\mathrm{B}$ & 12 & 73.64 & .000 & yes \\
Nonsymbolic approximate & $\mathrm{A}$ & 12 & 52.53 & .067 & no \\
comparison & $\mathrm{B}$ & 12 & 76.04 & .000 & yes \\
\hline
\end{tabular}

${ }^{\mathrm{c}}$ Continuous quantity conditions: $\mathrm{A}=\mathrm{dot}$ size, total dot surface area, total dot contour length and density positively correlated with number while array size negatively correlated with number; $\mathrm{B}=$ dot size, total dot surface area, total dot contour length and density negatively correlated with number while array size positively correlated with number. 


\section{KINDERGARTEN MATH ACHIEVEMENT}

chance level in both conditions. In the case of the comparison task, they performed above chance in condition B. In condition A this was not the case, however, children did not perform significantly below chance level and therefore we cannot assume that their responses were reliant on this condition's continuous quantity variables. 


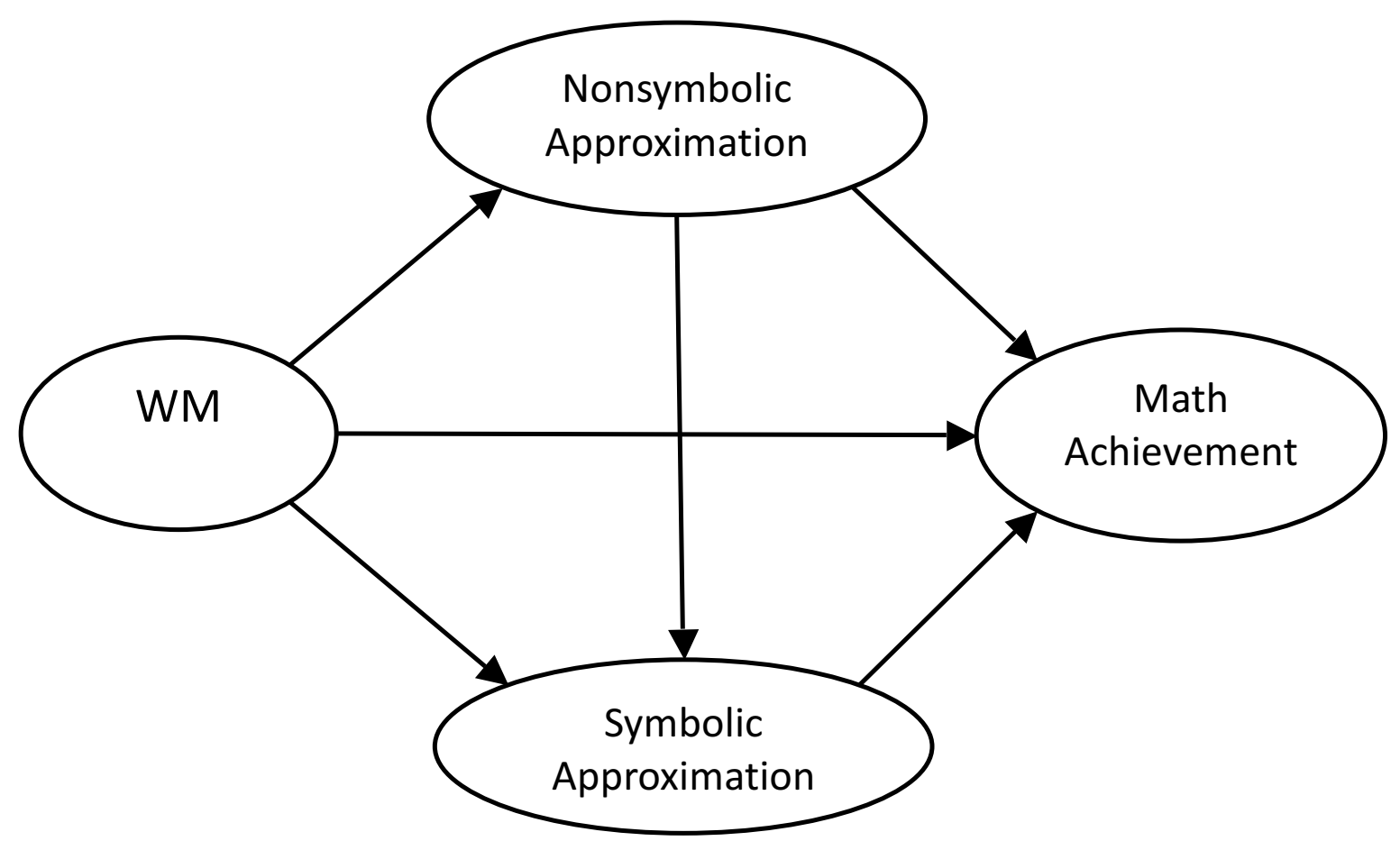

Figure 1. The integrative theoretical model association between Working Memory (WM), nonsymbolic, symbolic approximation skills and mathematics achievement in kindergarten. 


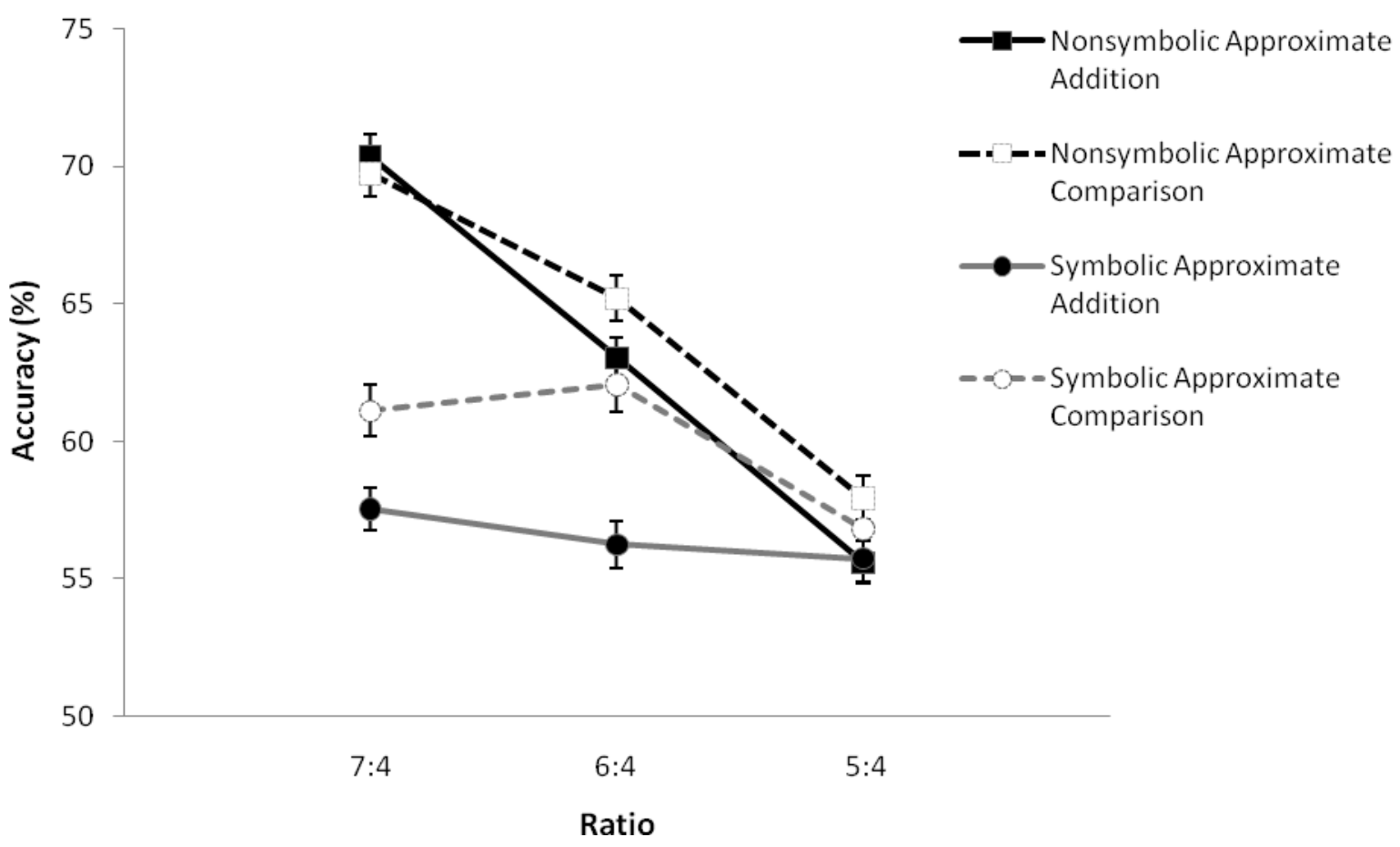

Figure 2. Ratio effects in children's accuracy on the nonsymbolic approximate addition, the nonsymbolic approximate comparison, the symbolic approximate addition and the symbolic approximate comparison task. The ratio effect was significant in each task, except for the symbolic addition task. 


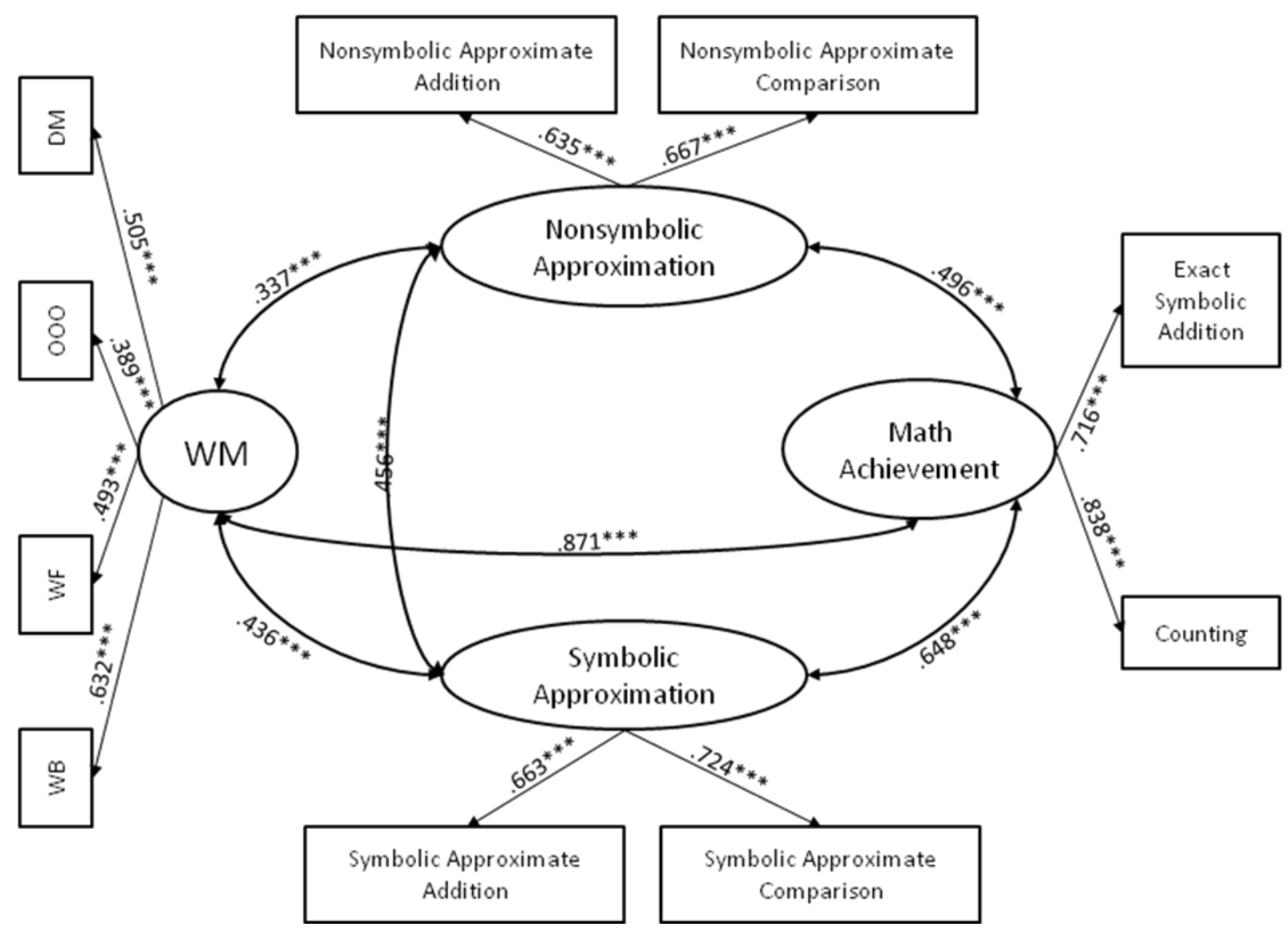

Figure 3. The CFA2 measurement model of WM, nonsymbolic and symbolic approximation and math achievement. Note: $\mathrm{WB}$ - word recall backwards, WF - word recall forward, $\mathrm{OOO}$ - odd one out, DM - cross matrix. $* * * p<.001$ 


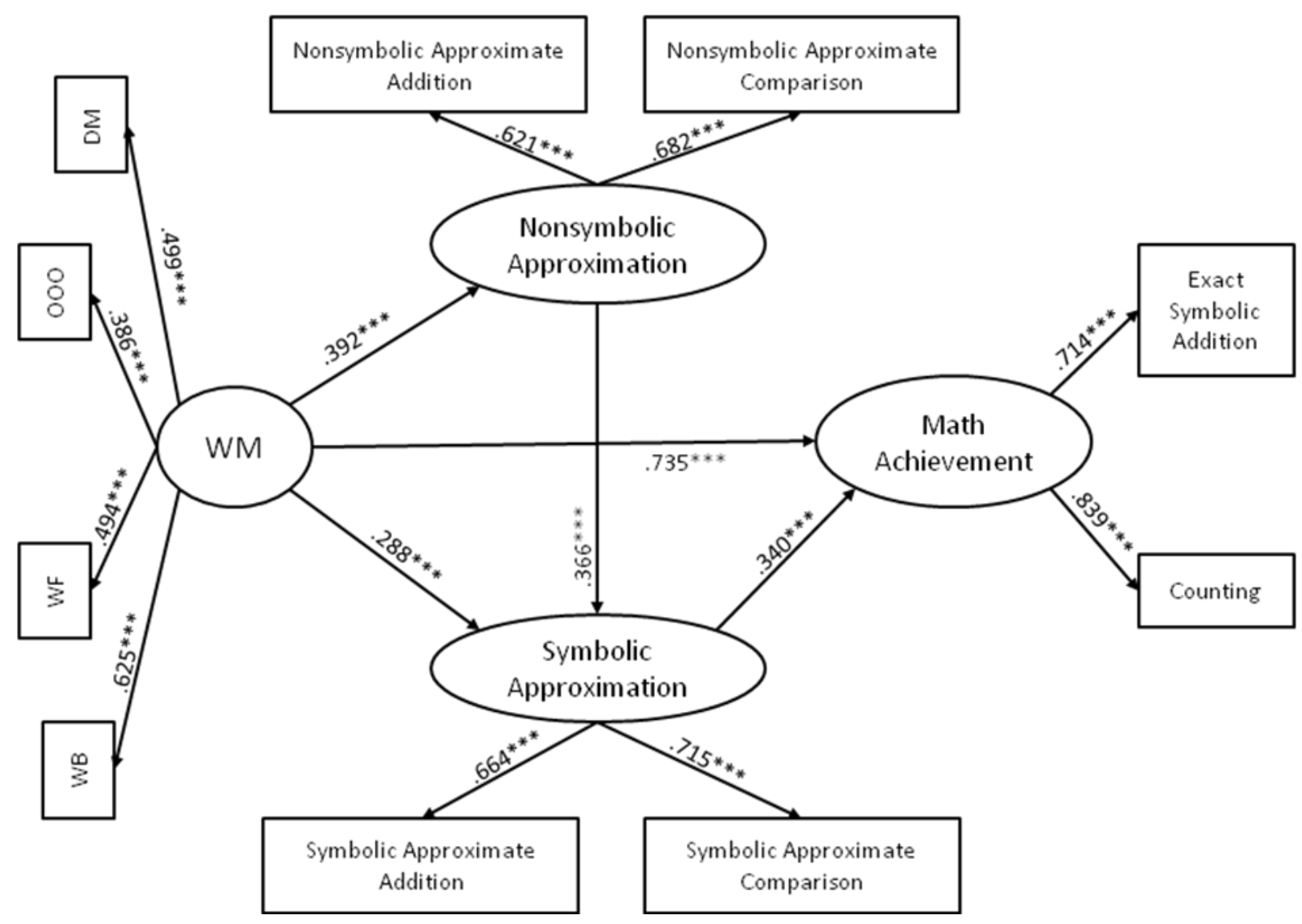

Figure 4. The Complete Mediation model of effects of nonsymbolic and symbolic approximation on math achievement. This was the most parsimonious and best fitting model. In the depicted model, $87.2 \%$ ( $S E=.064$, $p<.001)$ of the variance of kindergarteners' math achievement was explained. Note: WB - word recall backwards, WF - word recall forward, OOO - odd one out, DM - cross matrix. ${ }^{* * *} p<.001$. The mediation term does not regard the WM latent variable, only the relationship between nonsymbolic, symbolic approximation and math achievement. 


\section{KINDERGARTEN MATH ACHIEVEMENT}

Table 1

Descriptive and Probability Distribution Statistics on our Measures

\begin{tabular}{|c|c|c|c|c|c|c|c|c|}
\hline Variables & $N$ & $M$ & $S D$ & Min & $\operatorname{Max}$ & Th.Max & Skewness & Kurtosis \\
\hline \multicolumn{9}{|l|}{ Working Memory } \\
\hline Word recall forward & 444 & 12.89 & 2.63 & 3 & 20 & 20 & -0.05 & 1.92 \\
\hline Cross Matrix & 443 & 9.87 & 2.8 & 0 & 16 & 20 & -0.31 & 0.14 \\
\hline Word recall backward & 443 & 4.40 & 1.90 & 0 & 10 & 20 & -0.17 & 0.99 \\
\hline Odd one out & 444 & 8.39 & 2.88 & 1 & 15 & 20 & 0.08 & -0.85 \\
\hline \multicolumn{9}{|l|}{ Nonsymbolic approximation } \\
\hline Addition & 441 & 15.12 & 2.61 & 7 & 23 & 24 & 0.08 & 0.33 \\
\hline Comparison & 441 & 15.43 & 3.20 & 8 & 23 & 24 & 0.22 & -0.59 \\
\hline \multicolumn{9}{|l|}{ Symbolic approximation } \\
\hline Addition & 440 & 13.56 & 2.83 & 4 & 23 & 24 & 0.61 & .67 \\
\hline Comparison & 440 & 14.40 & 3.65 & 6 & 24 & 24 & 0.49 & -.02 \\
\hline \multicolumn{9}{|l|}{ Math Achievement } \\
\hline Counting & 443 & 10.77 & 4.49 & 0 & 20 & 20 & -0.20 & -0.76 \\
\hline Exact Symbolic Addition & 443 & 5.45 & 4.51 & 0 & 15 & 15 & 0.29 & -1.13 \\
\hline
\end{tabular}

Note. Th.Max $=$ Theoretical maximum score. In our analyses missing data was handled in a pairwise manner. 
Table 2.

Correlations Between Performance in the Math Achievement (1-2), WM (3-6), Nonsymbolic (7-8) and Symbolic (9-10) Approximation Tasks.

\begin{tabular}{|c|c|c|c|c|c|c|c|c|c|}
\hline & 1 & 2 & 3 & 4 & 5 & 6 & 7 & 8 & 9 \\
\hline 1. Counting & & & & & & & & & \\
\hline 2. Exact Addition & $.60 * * *$ & & & & & & & & \\
\hline 3. Cross Matrix & $.39 * * *$ & $.36 * * *$ & & & & & & & \\
\hline 4. Odd One Out & $.32 * * *$ & $.18^{* * *}$ & $.24 * * *$ & & & & & & \\
\hline 5. Word Recall Forward & $.37 * * *$ & $.26^{* * *}$ & $.20 * * *$ & $.13^{* *}$ & & & & & \\
\hline 6. Word Recall Backwards & $.46 * * *$ & $.36 * * *$ & $.28^{* * *}$ & $.25^{* * *}$ & $.39 * * *$ & & & & \\
\hline 7. Nonsymbolic Approximate Addition & $.26 * * *$ & $.25^{* * *}$ & .04 & .06 & $.13^{* *}$ & $.13^{* *}$ & & & \\
\hline 8. Nonsymbolic Approximate Comparison & $.26 * * *$ & $.26^{* * *}$ & $.15^{* *}$ & .08 & $.16^{* * *}$ & $.13^{* *}$ & $.42 * * *$ & & \\
\hline 9. Symbolic Approximate Addition & $.32 * * *$ & $.33^{* * *}$ & $.19 * * *$ & .05 & $.16^{* * *}$ & $.17^{* * *}$ & $.26^{* * *}$ & $.24 * * *$ & \\
\hline 10. Symbolic Approximate Comparison & $.39 * * *$ & $.39 * * *$ & $.23 * * *$ & $.15^{* * *}$ & .09 & $.19 * * *$ & $.14^{* *}$ & $.20 * * *$ & $.48^{* * *}$ \\
\hline
\end{tabular}

$* * * p \leq .001, * * p \leq .01$ 
Fit Statistics on the Structural Equation Models and the Corresponding Fit Criteria.

\begin{tabular}{|c|c|c|c|c|c|c|c|c|}
\hline Model & & & & & ices & & & \\
\hline Measurement & $x^{2}$ & $d f$ & $\chi^{2} / d f$ & CFI & $T L I$ & RMSEA & SRMR & \\
\hline CFA 1 & 114.69 & 32 & 3.58 & 0.910 & 0.873 & 0.076 & 0.048 & \\
\hline CFA 2 & 57.605 & 29 & 1.99 & 0.969 & 0.951 & 0.047 & 0.033 & \\
\hline SEM & & & & & & & & $\Delta \mathrm{x}^{2}$ \\
\hline Partial Mediation & 57.605 & 29 & 1.99 & 0.969 & 0.951 & 0.047 & 0.033 & - \\
\hline Direct Effects & 74.244 & 30 & 2.47 & 0.952 & 0.927 & 0.058 & 0.034 & $16.64 * * *$ \\
\hline $\begin{array}{l}\text { Complete } \\
\text { Mediation }\end{array}$ & 60.674 & 30 & 2.02 & 0.966 & 0.950 & 0.048 & 0.046 & $p=.08$ \\
\hline Acceptable fit & & & $\leq 5.0$ & $\geq .90$ & $\geq .90$ & $<.08$ & $\leq .10$ & \\
\hline Good fit & & & $0 \leq \chi^{2} / \mathrm{df} \leq 2$ & $\geq .95$ & $\geq .95$ & $<.05$ & $.00 \leq \mathrm{SRMR} \leq$ & \\
\hline
\end{tabular}

Note. $\chi^{2}=$ chi-square value; $d f=$ degrees of freedom; $\chi^{2} / d f=$ chi-square by degrees of freedom ratio; $C F I=$ Comparative Fir Index; $T L I=$ Tucker-Lewis Index; RMSEA = Root Mean Square Error Approximation; SRMR = Standardized Root Mean Square Residual; $A I C=\mathrm{Akaike}$ Information Criterion; $B I C=$ Bayesian Information Criterion; $S A B I C=$ Sample-size Adjusted BIC; $\Delta \chi^{2}=$ chi-square difference test: The Partial Mediation model was compared to the Direct Effects and the Complete Mediation model (1 degree of freedom for all comparisons). The mediation terms in the SEM models do not regard the WM latent variable, only the relationship between nonsymbolic, symbolic approximation and math achievement.

$* * * p<.001$ 